RUP-15-24

\title{
High efficiency of collisional Penrose process requires heavy particle production
}

\author{
${ }^{1}$ Kota Ogasawara, ${ }^{*}{ }^{1}$ Tomohiro Harada, ${ }^{\dagger}$ and ${ }^{2}$ Umpei Miyamoto $^{\ddagger}$ \\ ${ }^{1}$ Department of Physics, Rikkyo University, \\ Toshima, Tokyo 171-8501, Japan and \\ ${ }^{2}$ RECCS, Akita Prefectural University, Akita 015-0055, Japan
}

(Dated: August 28, 2018)

\begin{abstract}
The center-of-mass energy of two particles can become arbitrarily large if they collide near the event horizon of an extremal Kerr black hole, which is called the Bañados-Silk-West (BSW) effect. We consider such a high-energy collision of two particles which started from infinity and follow geodesics in the equatorial plane and investigate the energy extraction from such a highenergy particle collision and the production of particles in the equatorial plane. We analytically show that, on the one hand, if the produced particles are as massive as the colliding particles, the energy-extraction efficiency is bounded by 2.19 approximately. On the other hand, if a very massive particle is produced as a result of the high-energy collision, which has negative energy and necessarily falls into the black hole, the upper limit of the energy-extraction efficiency is increased to $(2+\sqrt{3})^{2} \simeq 13.9$. Thus, higher efficiency of the energy extraction, which is typically as large as 10, provides strong evidence for the production of a heavy particle.
\end{abstract}

PACS numbers: 04.70.Bw,97.60.Lf

\footnotetext{
*k.ogasawara@rikkyo.ac.jp

$\dagger$ harada@rikkyo.ac.jp

$\ddagger$ umpei@akita-pu.ac.jp
} 


\section{INTRODUCTION}

Bañados, Silk, and West (BSW) pointed out that the center-of-mass (CM) energy of two colliding particles can be arbitrarily large, if the collision occurs near the event horizon of an extremal Kerr black hole and the angular momentum of either of the colliding particles is finetuned to the critical value [1]. This is now called the BSW effect. See Harada and Kimura [2] for a brief review and references therein for further details. Particle collision with high CM energy had already been noticed by Piran, Shaham, and Katz in the study of energy extraction from collisional events in the ergoregion, which is called collisional Penrose process $[3,4]$. This process is typically as follows. We consider the reaction of particles 1 and 2 into particles 3 and 4 in the ergoregion, where particle 3 escapes to the infinity after the collision, while particle 4 falls into the black hole possibly with negative energy due to the existence of the ergosphere. If one defines an energy-extraction efficiency as

$$
\eta:=\frac{\text { energy of the escaping particle }}{\text { total energy of the injected particles }}=\frac{E_{3}}{E_{1}+E_{2}}
$$

the energy extraction $(\eta>1)$ from the black hole is possible provided $E_{4}<0$.

The high-CM-energy collision can produce a very massive and/or energetic particle. This means that Kerr black holes act as natural particle accelerators, which can accelerate even neutral particles. Recently, the interplay between such particle acceleration and energy extraction have been intensively investigated [5-8]. In particular, Schnittman [6] numerically showed that the upper limit of energy-extraction efficiency in the collisional Penrose process can reach about 13.9. Berti, Brito, and Cardoso confirmed the result of Ref. [6], and also showed that an arbitrarily high efficiency is possible by more general processes. Namely, they considered a head-on collision of two subcritical particles, which is called super-Penrose process. This process needs an outgoing particle which must be generated in the ergoregion by some preceding process $[7,8]$.

In this paper, we study particle collision near the horizon of an extremal Kerr black hole and the resultant energy extraction. We present an analytic formulation to investigate collisional Penrose process under the assumption that particles follow geodesics in the equatorial plane and two particles collide near the horizon to produce two particles. We find that if the produced particles are as massive as the colliding particles, the energy-extraction efficiency is bounded by 2.19 approximately. However, if a very massive particle is allowed to 
be produced, which has negative energy and necessarily falls into the black hole, the upper limit is increased to $(2+\sqrt{3})^{2} \simeq 13.9$, confirming the numerical result in Ref. [6].

The organization of this paper is as follows. In Sec. II, we prepare for the analysis of collisional Penrose process, reviewing geodesic motions and near-horizon collision in the Kerr black hole. In Sec. III A, we investigate the upper limits of the energy of escaping particle and of the energy-extraction efficiency in the case of produced particles as massive as the colliding particles. In Sec. III B, we will see that how the upper limits will be significantly increased if we take the production of a very massive particles due to the BSW effect into account. Section IV is devoted to conclusion. We adopt the geometrized unit in which $c=G=1$.

\section{PRELIMINARIES}

\section{A. Geodesics in the Kerr black hole}

The spacetime metric of the Kerr black hole is given by

$$
\begin{aligned}
g_{\mu \nu} d x^{\mu} d x^{\nu}=-\left(1-\frac{2 M r}{\rho^{2}}\right) d t^{2}- & \frac{4 M a r \sin ^{2} \theta}{\rho^{2}} d t d \varphi+\frac{\rho^{2}}{\Delta} d r^{2}+\rho^{2} d \theta^{2} \\
& +\left(r^{2}+a^{2}+\frac{2 M a^{2} r \sin ^{2} \theta}{\rho^{2}}\right) \sin ^{2} \theta d \varphi^{2},
\end{aligned}
$$

where $\rho^{2}(r, \theta):=r^{2}+a^{2} \cos ^{2} \theta, \Delta(r):=r^{2}-2 M r+a^{2}$ and $M$ and $a(0 \leq a \leq M)$ are the mass and spin parameters, respectively. $\Delta(r)$ vanishes at $r_{ \pm}:=M \pm \sqrt{M^{2}-a^{2}}$, and $r=r_{+}$ and $r=r_{-}$correspond to the event horizon and Cauchy horizon, respectively.

This spacetime is stationary and axisymmetric with Killing vectors $\partial_{t}$ and $\partial_{\varphi}$. The conserved energy $E$ and angular momentum $L$ of a particle with the four-momentum $p^{\mu}$ are given by $E=-g_{\mu \nu}\left(\partial_{t}\right)^{\mu} p^{\nu}=-g_{t \mu} p^{\mu}$ and $L=g_{\mu \nu}\left(\partial_{\varphi}\right)^{\mu} p^{\nu}=g_{\varphi \mu} p^{\mu}$, respectively. The components of the four-momentum are given in terms of these conserved charges as (e.g. [9, 10])

$$
\begin{aligned}
& p^{t}=\frac{1}{\Delta}\left[\left(r^{2}+a^{2}+\frac{2 M a^{2}}{r}\right) E-\frac{2 M a}{r} L\right], \quad p^{\varphi}=\frac{1}{\Delta}\left[\frac{2 M a}{r} E+\left(1-\frac{2 M}{r}\right) L\right], \quad \\
& \frac{1}{2}\left(p^{r}\right)^{2}+V=0, \quad V(r)=-\frac{M m^{2}}{r}+\frac{L^{2}-a^{2}\left(E^{2}-m^{2}\right)}{2 r^{2}}-\frac{M(L-a E)^{2}}{r^{3}}-\frac{E^{2}-m^{2}}{2},
\end{aligned}
$$


where $m(\geq 0)$ denotes the mass of the particle, and the motion is assumed to be confined in the equatorial plane, where $\theta=\pi / 2$.

The forward-in-time condition $p^{t}>0$ near the horizon $r \rightarrow r_{+}+0$ reduces to

$$
E-\Omega_{H} L \geq 0
$$

where $\Omega_{H}:=a /\left(r_{+}^{2}+a^{2}\right)$ is the angular velocity of the horizon. We call a particle a critical particle if it has a critical angular momentum $E / \Omega_{H}$, for which the equality in Eq. (2.4) holds. Accordingly, we call a particle with $L<E / \Omega_{H}\left(L>E / \Omega_{H}\right)$ a subcritical (supercritical) particle.

In the rest of this paper, we only consider the extremal black hole $a=M$. In this case, the forward-in-time condition for general position $r>r_{+}$is written as

$$
\frac{1}{2}\left[\left(\frac{r}{M}\right)^{3}+\frac{r}{M}+2\right] E>\tilde{L}
$$

where $\tilde{L}:=L / M$ is a reduced angular momentum.

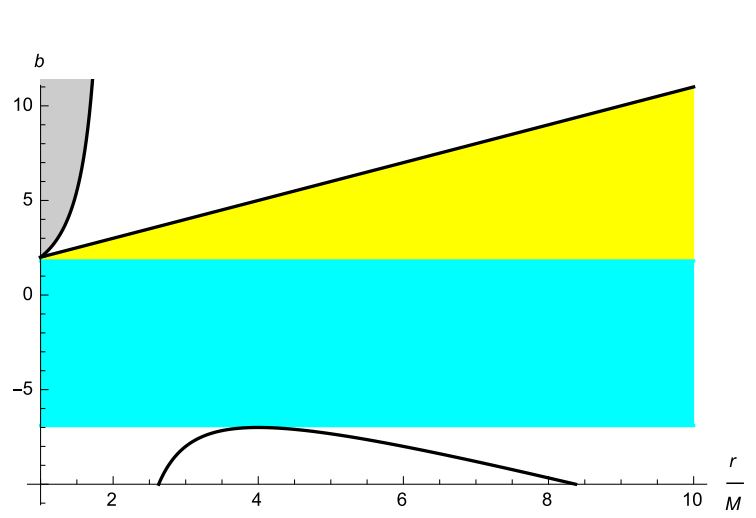

(a)

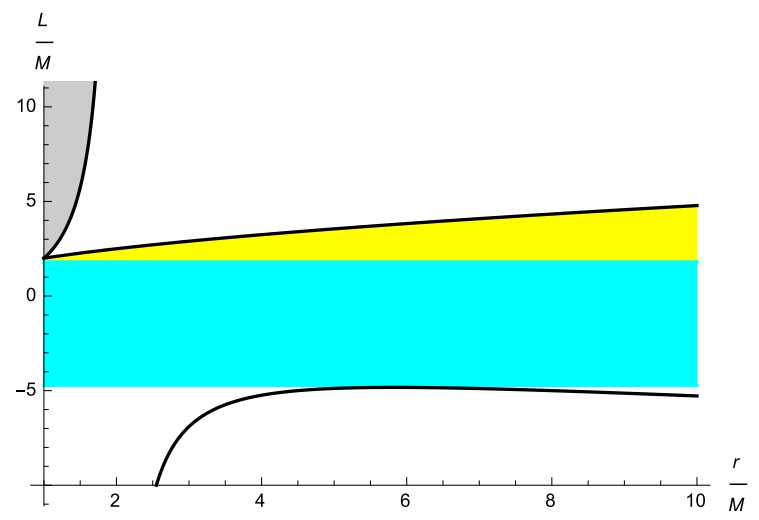

(b)

FIG. 1. (a) The radial turning points are plotted for a massless particle with $b=b_{ \pm}(r)$. (b) The radial turning points are plotted for a massive particle with $\tilde{L}=\tilde{L}_{ \pm}(r, E, m)$, where we set $E=m=1$. The negative energy particles are confined to the gray regions.

\section{B. Radial turning points of a geodesic particle}

Here, we are concerned with a particle that comes from or escapes to the infinity, which requires the effective potential $V$ in Eq. (2.3) is non-positive for large $r$. This requires 
$E \geq m$

For a massless particle $(m=0)$, solving $V=0$ for the impact parameter $b:=L / E$, we obtain $b=b_{ \pm}(r)$, where

$$
b_{+}(r):=r+M, \quad b_{-}(r):=-\left(r+M+\frac{4 M^{2}}{r-2 M}\right) .
$$

This means that a particle of which impact parameter $b=b_{ \pm}(r)$ has a turning point at $r$. The numerical plot of $b=b_{ \pm}(r)$ is given in Fig. 1(a). As $r$ increases from $M$ to infinity, $b_{+}(r)$ begins with $2 M$ and monotonically increases to infinity. As $r$ increases from $M$ to $2 M, b_{-}(r)$ begins with $2 M$ and monotonically increases to infinity. As $r$ increases from $2 M$ to infinity, $b_{-}(r)$ begins with negative infinity, monotonically increases to a local maximum $-7 M$ at $r=4 M$ and monotonically decreases to negative infinity. Therefore, for $2 M<b<b_{+}\left(r_{*}\right)$, the particle can escape to the infinity irrespective of the sign of the initial velocity, which is shown by the yellow region in Fig. 1(a) and where we denote the radial coordinate of collision by $r_{*}$. On the other hand, for $M<r_{*}<4 M$ and $-7 M<b \leq 2 M$, the particle can escape to the infinity only if it moves initially outwardly, which is shown by the blue region of Fig. 1(a).

For a massive particle $(m>0)$, solving $V(r)=0$ for $\tilde{L}$, we obtain $\tilde{L}=\tilde{L}_{ \pm}(r, E, m)$, where

$$
\tilde{L}_{ \pm}(r, E, m):=\frac{-2 M^{2} E \pm r(r-M) \sqrt{E^{2}-m^{2}+2 M m^{2} / r}}{M(r-2 M)}
$$

This means that a particle with $E, m$, and $\tilde{L}=\tilde{L}(r, E, m)$ has a turning point at $r$. The numerical plot of $\tilde{L}=\tilde{L}_{ \pm}(r, E, m)$ is given in Fig. 1(b). As $r$ increases from $M$ to infinity, $\tilde{L}_{+}(r, E, m)$ begins with $2 E$ and monotonically increases to infinity. As $r$ increases from $M$ to $2 M, \tilde{L}_{-}(r, E, m)$ begins with $2 E$ and monotonically increases to infinity. As $r$ increases from $2 M$ to infinity, $\tilde{L}_{-}(r, E, m)$ begins with negative infinity, monotonically increases to a local maximum $\tilde{L}_{\max }(E, m)(<0)$ at $r=r_{\max }$ and monotonically decreases to negative infinity. Therefore, for $2 E<\tilde{L}<\tilde{L}_{+}\left(r_{*}, E, m\right)$, the particle can escape to infinity irrespective of the sign of the initial velocity, which is shown by the yellow region of Fig. 1(b). On the other hand, for $M<r_{*}<r_{\max }$ and $\tilde{L}_{\max }(E, m)<\tilde{L} \leq 2 E$, the particle can escape to infinity only if it moves initially outwardly, which is shown by the blue region of Fig. 1(b). 


\section{Particle collision on the horizon}

Let us consider the reaction of two colliding particles, named particles 1 and 2, to two product particles, 3 and 4 . The local conservation of four-momenta can be written as

$$
p_{1}^{\mu}+p_{2}^{\mu}=p_{3}^{\mu}+p_{4}^{\mu}
$$

The $t$ - and $\varphi$-components of Eq. (2.8) represent the conservations of energy and angular momentum

$$
E_{1}+E_{2}=E_{3}+E_{4} \quad \text { and } \quad \tilde{L}_{1}+\tilde{L}_{2}=\tilde{L}_{3}+\tilde{L}_{4}
$$

respectively. The $r$-component represents the conservation of radial momentum

$$
\sigma_{1}\left|p_{1}^{r}\right|+\sigma_{2}\left|p_{2}^{r}\right|=\sigma_{3}\left|p_{3}^{r}\right|+\sigma_{4}\left|p_{4}^{r}\right|
$$

where $\sigma_{i}=\operatorname{sgn}\left(p_{i}^{r}\right)$ for $i=1,2,3,4$. Note that the mass and four-momentum of particle 4 can be written in terms of those of the other particles using the momentum conservations and identity $m_{4}^{2}=-p_{4}^{\mu} p_{4 \mu}$. From Eq. (2.3), we obtain

$$
\left|p_{i}^{r}\right|=2 E_{i}-\tilde{L}_{i}
$$

where we have used the forward-in-time condition to open the square root.

In the rest of this paper, we assume particle 1 to be critical $\left(\tilde{L}_{1}=2 E_{1}\right)$, particle 2 to be subcritical $\left(\tilde{L}_{2}<2 E_{2}\right)$, particle 3 to escape to infinity, and particle 4 to fall into the black hole with negative energy $\left(E_{4}<0\right)$. Then, since particle 1 is critical, Eq. (2.10) is written as

$$
\sigma_{2}\left(2 E_{2}-\tilde{L}_{2}\right)=\left\{\begin{array}{ll}
\sigma_{3}\left(2 E_{2}-\tilde{L}_{2}\right) & \left(\text { for } \sigma_{3}=\sigma_{4}\right) \\
\sigma_{3}\left[2\left(2 E_{3}-\tilde{L}_{3}\right)-\left(2 E_{2}-\tilde{L}_{2}\right)\right] & \left(\text { for } \sigma_{3}=-\sigma_{4}\right)
\end{array} .\right.
$$

When we choose $\sigma_{2}=-1$, several situations are possible depending on the values of $\sigma_{3}$ and $\sigma_{4}$. We will see, however, that only a few situations among them are interesting for our considerations. If $\sigma_{3}=\sigma_{4}=1$, from Eq. (2.12), we obtain $2 E_{2}-\tilde{L}_{2}=0$, which contradicts our assumption. If $\sigma_{3}=\sigma_{4}=-1$, we obtain $2 E_{2}-\tilde{L}_{2}=2 E_{3}-\tilde{L}_{3}+2 E_{4}-\tilde{L}_{4}$, which implies that particle 3 can be either critical $\left(\tilde{E}_{3}=2 E_{3}\right)$ or subcritical $\left(\tilde{E}_{3}<2 E_{3}\right)$. Nevertheless, only the critical case is interesting since a subcritical ingoing particle cannot escape to infinity. If $\sigma_{3}=-\sigma_{4}=1$, we obtain $2 E_{3}-\tilde{L}_{3}=0$ (particle 3 is critical). If 
$\sigma_{3}=-\sigma_{4}=-1$, we obtain $2 E_{2}-\tilde{L}_{2}=2 E_{3}-\tilde{L}_{3}$, which implies particle 3 is subcritical, it is not interesting since a subcritical ingoing particle cannot escape to infinity again.

When we choose $\sigma_{2}=1$, only a few situations are interesting again. If $\sigma_{3}=\sigma_{4}=1$, we obtain $2 E_{2}-\tilde{L}_{2}=\left(2 E_{3}-\tilde{L}_{3}\right)+\left(2 E_{4}-\tilde{L}_{4}\right)$, which implies that particle 3 can be either critical or subcritical. Since $\sigma_{3}=1$ in the present case, particle 3 can escape to infinity even if it is subcritical and outgoing, provided $b_{3}$ or $\tilde{L}_{3}$ satisfy $b_{\max , 3}<b_{3}$ (massless case) or $\tilde{L}_{\max , 3}\left(E_{3}, m_{3}\right)<\tilde{L}_{3}$ (massive case). If $\sigma_{3}=\sigma_{4}=-1$, we obtain $2 E_{2}-\tilde{L}_{2}=0$, which contradicts our assumption. If $\sigma_{3}=-\sigma_{4}=1$, we obtain $2 E_{2}-\tilde{L}_{2}=2 E_{3}-\tilde{L}_{3}$, which implies that particle 3 is subcritical. If $\sigma_{3}=-\sigma_{4}=-1$, we obtain $2 E_{3}-\tilde{L}_{3}=0$.

From the above considerations, we see the following four situations are interesting for the energy efficiency. Case A: $\sigma_{2}=-1$ and particle 2 is subcritical. In this case, particles 1 and 2 come from infinity and particle 3 is critical at the horizon. Case B: $\sigma_{2}=1$ and particle 2 is subcritical. In this case, particle 2 must be created inside the ergoregion by some preceding process. In the Appendix, we will see that the upper limit of the energy extraction efficiency is in case B is the same as in case A. Hence, we will focus on case A in Secs. III A and III B.

\section{Near-horizon and near-critical behaviors of a particle}

We parameterize the radial position of near-horizon collision $r_{*}$ as

$$
r_{*}=\frac{M}{1-\epsilon}, \quad 0<\epsilon \ll 1,
$$

and the near-critical angular momentum as

$$
\tilde{L}=2 E(1+\delta), \quad|\delta| \ll 1 .
$$

Then, we assume that $\delta$ can be expanded in powers of $\epsilon$ as

$$
\delta=\delta_{(1)} \epsilon+\delta_{(2)} \epsilon^{2}+O\left(\epsilon^{3}\right) .
$$

Under the assumption that particle 3 escapes to the infinity, $\tilde{L}_{3} \leq \tilde{L}_{+}\left(r_{*}, E_{3}, m_{3}\right)$ has to hold, which implies

$$
\delta \leq\left(\frac{2 E_{3}-\sqrt{E_{3}^{2}+m_{3}^{2}}}{2 E_{3}}\right) \epsilon+\left(\frac{4 E_{3} \sqrt{E_{3}^{2}+m_{3}^{2}}-3 E_{3}^{2}-2 m_{3}^{2}}{2 E_{3} \sqrt{E_{3}^{2}+m_{3}^{2}}}\right) \epsilon^{2}+O\left(\epsilon^{3}\right) .
$$

The forward-in-time condition for the near-horizon and near-critical particle implies

$$
\delta<\epsilon+\frac{7}{4} \epsilon^{2}+O\left(\epsilon^{3}\right)
$$

Therefore, the forward-in-time condition is always satisfied. 


\section{E. Expansion of $\left|p_{i}^{r}\right|$ by $\epsilon$}

Let us consider the series expansion of the radial momentum in powers of $\epsilon$ for each particle. Since we have assumed particle 1 to be critical and particle 2 to be subcritical, $\left|p_{1}^{r}\right|$ and $\left|p_{2}^{r}\right|$ are expanded as

$$
\begin{aligned}
& \left|p_{1}^{r}\right|=\sqrt{3 E_{1}^{2}-m_{1}^{2}} \epsilon-\frac{E_{1}^{2}}{\sqrt{3 E_{1}^{2}-m_{1}^{2}}} \epsilon^{2}+O\left(\epsilon^{3}\right), \\
& \left|p_{2}^{r}\right|=\left(2 E_{2}-\tilde{L}_{2}\right)-2\left(E_{2}-\tilde{L}_{2}\right) \epsilon+\frac{\left(3 E_{2}-\tilde{L}_{2}\right)\left(E_{2}-\tilde{L}_{2}\right)-m_{2}^{2}}{2\left(2 E_{2}-\tilde{L}_{2}\right)} \epsilon^{2}+O\left(\epsilon^{3}\right) .
\end{aligned}
$$

If particle 3 is near critical and particle 4 is subcritical, the expansions of $\left|p_{3}^{r}\right|$ and $\left|p_{4}^{r}\right|$ are given by

$$
\begin{aligned}
&\left|p_{3}^{r}\right|= \sqrt{E_{3}^{2}\left[4\left(1-\delta_{(1)}\right)^{2}-1\right]-m_{3}^{2}} \epsilon-\frac{E_{3}^{2}\left[1-4\left(2 \delta_{(1)}-\delta_{(2)}\right)\left(1-\delta_{(1)}\right)\right]}{\sqrt{E_{3}^{2}\left[4\left(1-\delta_{(1)}\right)^{2}-1\right]-m_{3}^{2}}} \epsilon^{2}+O\left(\epsilon^{3}\right), \\
&\left|p_{4}^{r}\right|=\left(2 E_{2}-\tilde{L}_{2}\right)-\left[2\left(E_{2}-\tilde{L}_{2}\right)+2 E_{3}\left(1-\delta_{(1)}\right)-2 E_{1}\right] \epsilon \\
&+\left[\frac{\left(2 E_{2}-\tilde{L}_{2}\right)}{2}-2 E_{3}\left(2 \delta_{(1)}-\delta_{(2)}\right)-\frac{\left(E_{1}+E_{2}-E_{3}\right)^{2}+m_{4}^{2}}{2\left(2 E_{2}-\tilde{L}_{2}\right)}\right] \epsilon^{2}+O\left(\epsilon^{3}\right) .
\end{aligned}
$$

If particle 3 is subcritical and particle 4 is near critical, the expansion of $\left|p_{3}^{r}\right|$ and $\left|p_{4}^{r}\right|$ are

obtained by exchanging subscripts 3 and 4 in Eqs. (2.20) and (2.21). If both particles 3 and 4 are subcritical, the expansion of $\left|p_{3}^{r}\right|$ and $\left|p_{4}^{r}\right|$ are given by

$$
\begin{aligned}
\left|p_{3}^{r}\right|= & \left(2 E_{3}-\tilde{L}_{3}\right)-2\left(E_{3}-\tilde{L}_{3}\right) \epsilon+\frac{\left(3 E_{3}-\tilde{L}_{3}\right)\left(E_{3}-\tilde{L}_{3}\right)-m_{3}^{2}}{2\left(2 E_{3}-\tilde{L}_{3}\right)} \epsilon^{2}+O\left(\epsilon^{3}\right), \\
\left|p_{4}^{r}\right|= & \left(2 E_{2}-\tilde{L}_{2}\right)-\left(2 E_{3}-\tilde{L}_{3}\right)+2\left(E_{1}-E_{2}+E_{3}+\tilde{L}_{2}-\tilde{L}_{3}\right) \epsilon \\
& +\frac{\left(E_{1}-E_{2}+E_{3}+\tilde{L}_{2}-\tilde{L}_{3}\right)\left(E_{1}+3 E_{2}-3 E_{3}-\tilde{L}_{2}+\tilde{L}_{3}\right)+m_{4}^{2}}{2\left[\left(2 E_{2}-\tilde{L}_{2}\right)-\left(2 E_{3}-\tilde{L}_{3}\right)\right]} \epsilon^{2}+O\left(\epsilon^{3}\right) .
\end{aligned}
$$

\section{ENERGY-EXTRACTION EFFICIENCY}

\section{A. Case for $m_{4}=O\left(\epsilon^{0}\right)$}

We focus on case A, where $\sigma_{2}=\sigma_{4}=-1$ and particle 3 is near critical. $\sigma_{2}=-1$ implies that we can assume that particles 1 and 2 come from the infinity. The $O(\epsilon)$ and $O\left(\epsilon^{2}\right)$ terms of radial momentum conservation Eq. (2.10) yield

$$
\sigma_{1} \sqrt{3 E_{1}^{2}-m_{1}^{2}}+2 E_{1}-2 E_{3}\left(1-\delta_{(1)}\right)=\sigma_{3} \sqrt{E_{3}^{2}\left[4\left(1-\delta_{(1)}\right)^{2}-1\right]-m_{3}^{2}},
$$


and

$$
\begin{array}{r}
\sigma_{1} \frac{E_{1}^{2}}{\sqrt{3 E_{1}^{2}-m_{1}^{2}}}+\frac{\left(3 E_{2}-\tilde{L}_{2}\right)\left(E_{2}-\tilde{L}_{2}\right)-m_{2}^{2}}{2\left(2 E_{2}-\tilde{L}_{2}\right)}=\sigma_{3} \frac{E_{3}^{2}\left[1-4\left(2 \delta_{(1)}-\delta_{(2)}\right)\left(1-\delta_{(1)}\right)\right]}{\sqrt{E_{3}^{2}\left[4\left(1-\delta_{(1)}\right)^{2}-1\right]-m_{3}^{2}}} \\
+\frac{\left(2 E_{2}-\tilde{L}_{2}\right)}{2}-2 E_{3}\left(2 \delta_{(1)}-\delta_{(2)}\right)-\frac{\left(E_{1}+E_{2}-E_{3}\right)^{2}+m_{4}^{2}}{2\left(2 E_{2}-\tilde{L}_{2}\right)}
\end{array}
$$

respectively.

When we choose $\sigma_{1}=1$, Eq. (3.1) implies

$$
B_{1}-2 E_{3}\left(1-\delta_{(1)}\right)=\sigma_{3} \sqrt{E_{3}^{2}\left[4\left(1-\delta_{(1)}\right)^{2}-1\right]-m_{3}^{2}},
$$

where $B_{1}:=2 E_{1}+\sqrt{3 E_{1}^{2}-m_{1}^{2}}(>0)$. Squaring the both sides of Eq. (3.3), we obtain

$$
1-\delta_{(1)}=\frac{B_{1}^{2}+E_{3}^{2}+m_{3}^{2}}{4 B_{1} E_{3}}
$$

which implies

$$
\delta_{(1), \max }-\delta_{(1)}=\frac{\left(B_{1}-\sqrt{E_{3}^{2}+m_{3}^{2}}\right)^{2}}{4 B_{1} E_{3}} \geq 0 .
$$

Substituting Eq. (3.4) into the left-hand side of Eq. (3.3), we obtain

$$
B_{1}-\frac{E_{3}^{2}+m_{3}^{2}}{B_{1}}=2 \sigma_{3} \sqrt{E_{3}^{2}\left[4\left(\delta_{(1)}-1\right)^{2}-1\right]-m_{3}^{2}} .
$$

This implies $E_{3} \leq \tilde{\lambda}_{0}:=\sqrt{B_{1}^{2}-m_{3}^{2}}\left(E_{3} \geq \tilde{\lambda}_{0}\right)$ for $\sigma_{3}=1\left(\sigma_{3}=-1\right)$.

If we choose $\sigma_{3}=-1$, we need $\delta_{(1)} \geq 0$ since particle 3 has to be scattered by the potential barrier. Supposing $\delta_{(1)} \geq 0$ in Eq. (3.4), we have

$$
E_{3}^{2}-4 B_{1} E_{3}+B_{1}^{2}+m_{3}^{2} \leq 0 .
$$

Inequality (3.7) is satisfied by

$$
\tilde{\lambda}_{-} \leq E_{3} \leq \tilde{\lambda}_{+}, \quad \tilde{\lambda}_{ \pm}:=2 B_{1} \pm \sqrt{3 B_{1}^{2}-m_{3}^{2}}
$$

where the discriminant $D$ of Eq. (3.7) has to satisfy $D / 4=3 B_{1}^{2}-m_{3}^{2} \geq 0$. Since particle 3 escapes to infinity, it is marginally bound or unbound $\left(E_{3} \geq m_{3}\right) . m_{3} \leq \tilde{\lambda}_{+}$has to be satisfied so that Eq. (3.8) and $E_{3} \geq m_{3}$ have an intersection. The relation $m_{3} \leq \tilde{\lambda}_{+}$is satisfied if $m_{3} \leq \sqrt{3} B_{1}$, which is equivalent to $D / 4 \geq 0$. Therefore, if $D / 4 \geq 0$ is satisfied, Eq. (3.8) and $E_{3} \geq m_{3}$ always have an intersection.

$\tilde{\lambda}_{0}$ and $\tilde{\lambda}_{+}$are the upper limits on $E_{3}$ for $\sigma_{3}=1$ and $\sigma_{3}=-1$, respectively. Since $\tilde{\lambda}_{+}$is larger than $\tilde{\lambda}_{0}$, we concentrate on the case of $\sigma_{3}=-1$. The maximum of $\tilde{\lambda}_{+}$is given by

$$
\tilde{\lambda}_{+, \max }=(2+\sqrt{3})^{2} E_{1},
$$


where we have assumed $m_{1}=m_{3}=0$.

Next, we consider the $O\left(\epsilon^{2}\right)$ terms in the radial momentum conservation. $E_{3}=\tilde{\lambda}_{+}$can be realized when $\delta_{(1)}=0$. Substituting $\delta_{(1)}=0, \sigma_{1}=1$, and $\sigma_{3}=-1$ into Eq. (3.2), and then solving it for $m_{4}^{2}$, we obtain

$$
\begin{aligned}
& m_{4}^{2}=-2\left(2 E_{2}-\tilde{L}_{2}\right)\left[\frac{E_{1}^{2}}{\sqrt{3 E_{1}^{2}-m_{1}^{2}}}+\frac{\tilde{\lambda}_{+}^{2}}{\sqrt{3 \tilde{\lambda}_{+}^{2}-m_{3}^{2}}}+\frac{2 \delta_{(2)} \tilde{\lambda}_{+}}{\left.\sqrt{3 \tilde{\lambda}_{+}^{2}-m_{3}^{2}}\left(2 \tilde{\lambda}_{+}-\sqrt{3 \tilde{\lambda}_{+}^{2}-m_{3}^{2}}\right)\right]}\right. \\
&+\left(E_{2}^{2}+m_{2}^{2}\right)-\left(E_{1}+E_{2}-\tilde{\lambda}_{+}\right)^{2} .
\end{aligned}
$$

We need $\delta>0$ for particle 3 to escape to the infinity. Since we choose $\delta_{(1)}=0$, we need $\delta_{(2)} \geq 0$. This implies the first term on the right-hand side of Eq. (3.10) is negative. The lower limit of $E_{2}$ is then given by

$$
E_{2} \geq \tilde{\kappa}:=\frac{1}{2}\left[\left(\tilde{\lambda}_{+}-E_{1}\right)-\frac{m_{2}^{2}}{\left(\tilde{\lambda}_{+}-E_{1}\right)}\right] .
$$

Since we assume that particle 2 comes from infinity, it must be marginally bound or unbound $\left(E_{2} \geq m_{2}\right)$. Therefore, we have to compare $\tilde{\kappa}$ with $m_{2}$. If $\tilde{\kappa} \geq m_{2}$, i.e., $(\sqrt{2}-1)\left(\tilde{\lambda}_{+}-E_{1}\right) \geq$ $m_{2}$, the lower limit of $E_{2}$ is $\tilde{\kappa}$. Thus, we find

$$
\eta \leq \frac{\tilde{\lambda}_{+}}{E_{1}+\tilde{\kappa}}=\frac{2 \tilde{\lambda}_{+}\left(\tilde{\lambda}_{+}-E_{1}\right)}{\tilde{\lambda}_{+}^{2}-E_{1}^{2}-m_{2}^{2}}=: f\left(m_{2}\right) .
$$

One can see that $f\left(m_{2}\right)$ defined above begins with $2 \tilde{\lambda}_{+} /\left(\tilde{\lambda}_{+}+E_{1}\right)$ and monotonically increases to

$$
\frac{(3+2 \sqrt{2}) \tilde{\lambda}_{+}}{(2+\sqrt{2}) E_{1}+(1+\sqrt{2}) \tilde{\lambda}_{+}}=: g\left(\tilde{\lambda}_{+}\right)
$$

as $m_{2}$ increase from 0 to $(\sqrt{2}-1)\left(\tilde{\lambda}_{+}-E_{1}\right)$. Since $g\left(\tilde{\lambda}_{+}\right)$is a monotonically increasing function of $\tilde{\lambda}_{+}$, the maximum of $g\left(\tilde{\lambda}_{+}\right)$is given by

$$
g\left(\tilde{\lambda}_{+, \max }\right)=\frac{179+186 \sqrt{2}+88 \sqrt{3}+100 \sqrt{6}}{383} \simeq 2.19 .
$$

If $\tilde{\kappa} \leq m_{2}$, i.e., $(\sqrt{2}-1)\left(\tilde{\lambda}_{+}-E_{1}\right) \leq m_{2}$, the lower limit of $E_{2}$ is $m_{2}$. Thus, we find

$$
\eta \leq \frac{\tilde{\lambda}_{+}}{E_{1}+m_{2}}
$$

and easily notice that the right-hand side monotonically decreases as $m_{2}$ increase. For the above reason, the maximum of the right-hand side is about 2.19.

In summary, the upper limit of the energy extraction efficiency is $\eta_{\max } \simeq 2.19$, which is realized $\sigma_{1}=1, \sigma_{2}=\sigma_{3}=\sigma_{4}=-1, m_{1}=m_{3}=0$, and $\delta_{(1)}=0$. 
Next, let us consider the case of $\sigma_{1}=-1$. Equation (3.1) implies

$$
A_{1}-2 E_{3}\left(1-\delta_{(1)}\right)=\sigma_{3} \sqrt{E_{3}^{2}\left[4\left(1-\delta_{(1)}\right)^{2}-1\right]-m_{3}^{2}},
$$

where $A_{1}:=2 E_{1}-\sqrt{3 E_{1}^{2}-m_{1}^{2}}(>0)$. Performing the following replacement, an argument similar to that in the $\sigma_{1}=1$ case applies.

$$
\begin{aligned}
B_{1} \rightarrow A_{1} & :=2 E_{1}-\sqrt{3 E_{1}^{2}-m_{1}^{2}} \\
\tilde{\lambda}_{0} \rightarrow \lambda_{0} & :=\sqrt{A_{1}^{2}-m_{3}^{2}} \\
\tilde{\lambda}_{ \pm} \rightarrow \lambda_{ \pm} & :=2 A_{1} \pm \sqrt{3 A_{1}^{2}-m_{3}^{2}} \\
\tilde{\kappa} \rightarrow \kappa & :=\frac{1}{2}\left[\left(\lambda_{+}-E_{1}\right)-\frac{m_{2}^{2}}{\left(\lambda_{+}-E_{1}\right)}\right] .
\end{aligned}
$$

However, the value of the upper limit is different. Since $\lambda_{+}$is larger than $\lambda_{0}$, we concentrate on the case $\sigma_{3}=-1$. The maximum of $\lambda_{+}$is given by

$$
\lambda_{+, \max }=(2+\sqrt{3})(2-\sqrt{2}) E_{1},
$$

where we have assumed $m_{1}=E_{1}$ and $m_{3}=0$. We have seen that $E_{3}=\lambda_{+}$can be realized $\delta_{(1)}=0$. Substituting $\delta_{(1)}=0$ and $\sigma_{1}=\sigma_{3}=-1$ into Eq. (3.2), and then solving it for $m_{4}^{2}$, we obtain

$$
\begin{array}{r}
m_{4}^{2}=-2\left(2 E_{2}-\tilde{L}_{2}\right)\left[-\frac{E_{1}^{2}}{\sqrt{3 E_{1}^{2}-m_{1}^{2}}}+\frac{\lambda_{+}^{2}}{\sqrt{3 \lambda_{+}^{2}-m_{3}^{2}}}+\frac{2 \delta_{(2)} \lambda_{+}}{\sqrt{3 \lambda_{+}^{2}-m_{3}^{2}}}\left(2 \lambda_{+}-\sqrt{3 \lambda_{+}^{2}-m_{3}^{2}}\right)\right] \\
+\left(E_{2}^{2}+m_{2}^{2}\right)-\left(E_{1}+E_{2}-\lambda_{+}\right)^{2} .
\end{array}
$$

Again, we need $\delta>0$ for particle 3 to escape to infinity. Since we choose $\delta_{(1)}=0$, we need $\delta_{(2)} \geq 0$. In this case, we can prove the first term on the right-hand side of Eq. (3.18) is negative (See Ref. [5, Appendix B]). The lower limit of $E_{2}$ is given by

$$
E_{2} \geq \kappa:=\frac{1}{2}\left[\left(\lambda_{+}-E_{1}\right)-\frac{m_{2}^{2}}{\left(\lambda_{+}-E_{1}\right)}\right] .
$$

From the discussion similar to that in the case of $\sigma_{1}=1$, the upper limit of the energy extraction efficiency is also obtained as

$$
\eta_{\max }=\frac{2+\sqrt{2}+\sqrt{6}}{4} \simeq 1.47
$$

which is realized $\sigma_{1}=\sigma_{2}=\sigma_{3}=\sigma_{4}=-1, E_{1}=m_{1}, m_{3}=0, \delta_{(1)}=0$, and $m_{2} / m_{1} \simeq 0.491$. This reproduces the result of Ref. [5]. 


\section{B. Case for $m_{4}=O\left(\epsilon^{-1 / 2}\right)$}

In general, the CM energy of particles 1 and 2 is given by

$$
E_{\mathrm{cm}}^{2}=-\left(p_{1}^{\mu}+p_{2}^{\mu}\right)\left(p_{1 \mu}+p_{2 \mu}\right)
$$

For example, in the original BSW process [1], in which $\tilde{L}_{1}=2 E_{1}, \tilde{L}_{2}<2 E_{2}$ and $\sigma_{1}=\sigma_{2}=$ -1 , the leading term of the CM energy is

$$
E_{\mathrm{cm}}^{2} \simeq \frac{2\left(2 E_{2}-\tilde{L}_{2}\right)\left(2 E_{1}-\sqrt{3 E_{1}^{2}-m_{1}^{2}}\right)}{\epsilon} .
$$

In such a high-energy collision, the masses of the product particles (particles 3 and 4) can become large with the following restriction

$$
m_{3}+m_{4} \leq E_{\mathrm{cm}}
$$

Since $E_{\mathrm{cm}}$ for the collision between a critical particle and a subcritical particle is proportional to $\epsilon^{-1 / 2}$ for both a rear-end collision and head-on collision, we assume

$$
m_{4}=O\left(\epsilon^{-1 / 2}\right)
$$

Then, we assume that particle 4 is very massive as

$$
m_{4}^{2}=\frac{\mu_{4}}{\epsilon}+\nu_{4}
$$

where $\mu_{4}(>0)$ and $\nu_{4}$ are constants. In fact, we can write $m_{4}^{2}$ in terms of the quantities of the other particles, using the momentum conservation, as

$$
m_{4}^{2}=-\left(p_{1}^{\mu}+p_{2}^{\mu}-p_{3}^{\mu}\right)\left(p_{1 \mu}+p_{2 \mu}-p_{3 \mu}\right)=E_{\mathrm{cm}}^{2}+m_{3}^{2}+2\left(p_{1}^{\mu}+p_{2}^{\mu}\right) p_{3 \mu}
$$

where we have assumed $\tilde{L}_{3}=2 E_{3}(1+\delta)$. This implies

$$
\mu_{4}=2\left(2 E_{2}-\tilde{L}_{2}\right)\left[2 E_{1}-2 E_{3}\left(1-\delta_{(1)}\right)+\sigma_{1} \sqrt{3 E_{1}^{2}-m_{1}^{2}}-\sigma_{3} \sqrt{E_{3}^{2}\left[4\left(1-\delta_{(1)}\right)^{2}-1\right]-m_{3}^{2}}\right] .
$$

The expansion of radial momentum of particle 4 is given by

$$
\begin{aligned}
\left|p_{4}^{r}\right|= & \left(2 E_{2}-\tilde{L}_{2}\right)-\left[2\left(E_{2}-\tilde{L}_{2}\right)+2 E_{3}\left(1-\delta_{(1)}\right)-2 E_{1}+\frac{\mu_{4}}{2\left(2 E_{2}-\tilde{L}_{2}\right)}\right] \epsilon \\
& +F_{4, \epsilon^{2}} \epsilon^{2}+O\left(\epsilon^{3}\right)
\end{aligned}
$$


where

$$
\begin{aligned}
F_{4, \epsilon^{2}}:= & \frac{\left(2 E_{2}-\tilde{L}_{2}\right)}{2}-2 E_{3}\left(2 \delta_{(1)}-\delta_{(2)}\right)-\frac{\left(E_{1}+E_{2}-E_{3}\right)^{2}+\nu_{4}}{2\left(2 E_{2}-\tilde{L}_{2}\right)} \\
& +\frac{\left[E_{1}-E_{2}+\tilde{L}_{2}-E_{3}\left(1-\delta_{(1)}\right)\right] \mu_{4}}{\left(2 E_{2}-\tilde{L}_{2}\right)^{2}}-\frac{\mu_{4}^{2}}{2\left(2 E_{2}-\tilde{L}_{2}\right)^{3}} .
\end{aligned}
$$

This implies that the $O(\epsilon)$ and $O\left(\epsilon^{2}\right)$ terms of the radial momentum conservation yield Eq. (3.27) and

$$
\sigma_{1} \frac{E_{1}^{2}}{\sqrt{3 E_{1}^{2}-m_{1}^{2}}}+\frac{\left(3 E_{2}-\tilde{L}_{2}\right)\left(E_{2}-\tilde{L}_{2}\right)-m_{2}^{2}}{2\left(2 E_{2}-\tilde{L}_{2}\right)}-F_{4, \epsilon^{2}}=\sigma_{3} \frac{E_{3}^{2}\left[1+4\left(2 \delta_{(1)}-\delta_{(2)}\right)\left(\delta_{(1)}-1\right)\right]}{\sqrt{E_{3}^{2}\left[4\left(\delta_{(1)}-1\right)^{2}-1\right]-m_{3}^{2}}},
$$

respectively.

If we define

$$
C:=2 E_{1}+\sigma_{1} \sqrt{3 E_{1}^{2}-m_{1}^{2}}-\frac{\mu_{4}}{2\left(2 E_{2}-\tilde{L}_{2}\right)}>0,
$$

we can discuss the upper limit of $E_{3}$ in the way similar to that in Sec. III A. As we have already seen, we need $\sigma_{3}=-1$ and $\delta_{(1)} \geq 0$ to obtain the maximum of $E_{3}$. Setting $\delta_{(1)} \geq 0$ in Eq. (3.27), we have

$$
E_{3}^{2}-4 C E_{3}+C^{2}+m_{3}^{2} \leq 0
$$

This inequality is satisfied by

$$
\bar{\lambda}_{-} \leq E_{3} \leq \bar{\lambda}_{+}, \quad \bar{\lambda}_{ \pm}:=2 C \pm \sqrt{3 C^{2}-m_{3}^{2}} .
$$

The maximum value of $\bar{\lambda}_{+}$is given by

$$
\bar{\lambda}_{+, \max }=(2+\sqrt{3})^{2} E_{1}-\frac{(2+\sqrt{3}) \mu_{4}}{2\left(2 E_{2}-\tilde{L}_{2}\right)},
$$

where we have assume $m_{1}=m_{3}=0$ and $\sigma_{1}=1 . \quad E_{3}=\bar{\lambda}_{+}$is realized when $\delta_{(1)}=0$. Substituting $\delta_{(1)}=0, \sigma_{1}=1$, and $\sigma_{3}=-1$ into Eq. (3.30), and then solving it for $\nu_{4}$, we obtain

$$
\begin{aligned}
\nu_{4}= & -2\left(2 E_{2}-\tilde{L}_{2}\right)\left[\frac{E_{1}^{2}}{\sqrt{3 E_{1}^{2}-m_{1}^{2}}}+\frac{\bar{\lambda}_{+}^{2}}{\sqrt{3 \bar{\lambda}_{+}^{2}-m_{3}^{2}}}+\frac{2 \delta_{(2)} \bar{\lambda}_{+}}{\sqrt{3 \bar{\lambda}_{+}^{2}-m_{3}^{2}}}\left(2 \bar{\lambda}_{+}-\sqrt{3 \bar{\lambda}_{+}^{2}-m_{3}^{2}}\right)\right] \\
& +\left(E_{2}^{2}+m_{2}^{2}\right)-\left(E_{1}+E_{2}-\bar{\lambda}_{+}\right)^{2}+\frac{2\left(E_{1}-E_{2}+\tilde{L}_{2}-\bar{\lambda}_{+}\right) \mu_{4}}{\left(2 E_{2}-\tilde{L}_{2}\right)}-\frac{\mu_{4}^{2}}{\left(2 E_{2}-\tilde{L}_{2}\right)^{2}} .(3.35)
\end{aligned}
$$


In Sec. III A, we have used the above equation with $\mu_{4}=0$ and $\nu_{4}=m_{4}^{2} \geq 0$ to estimate the lower limit of $E_{2}$. However, in the present case, the sign of $\nu_{4}$ is not restricted to be positive if $\mu_{4}>0$. Hence, we use Eq. (3.35) not for the estimate of the lower limit of $E_{2}$ but for the determination of $\nu_{4}$. Therefore, the upper limit of the energy-extraction efficiency is given by

$$
\eta_{\max }=\frac{\bar{\lambda}_{+, \max }}{E_{1}+E_{2}}=\frac{(2+\sqrt{3})^{2} E_{1}-\frac{(2+\sqrt{3}) \mu_{4}}{2\left(2 E_{2}-\tilde{L}_{2}\right)}}{E_{1}+E_{2}} .
$$

From the above equation, we see that if $E_{1} E_{2} \gg \mu_{4}, \eta_{\max }$ is approximately given by $(2+$ $\sqrt{3})^{2} E_{1} /\left(E_{1}+E_{2}\right)$. Moreover, for $E_{2} / E_{1} \ll 1$, we find that the escaping massless particle has the energy that is nearly equal to $(2+\sqrt{3})^{2} \simeq 13.9$ times the total energy of the incoming massless particles.

However, $E_{3}$ cannot be exactly equal to $(2+\sqrt{3})^{2} E_{1}$, for the following reason. From Eq. (3.34), $E_{3}=(2+\sqrt{3})^{2} E_{1}$ is realized when $m_{1}=m_{3}=0, \delta_{(1)}=0$ and $\mu_{4}=0$. However, $\mu_{4}=0$ implies $m_{4}^{2}=O\left(\epsilon^{0}\right)$ and hence $\nu_{4}$ must be positive. Substituting $\mu_{4}=0$ into Eq. (3.35) and requiring $\nu_{4}>0$, we obtain the lower limit of $E_{2}$, which is given by Eq. (3.11). In this case, we have already seen that the efficiency cannot reach $(2+\sqrt{3})^{2} \simeq 13.9$ but only 2.19.

While there are other cases in which a very massive and/or energetic particle is produced, one can conclude that a large efficiency is possible only in the above case. In any other cases, $p_{3}^{r}$ and $p_{4}^{r}$ have $O\left(\epsilon^{1 / 2}\right)$ terms. Since there is no half-integer order terms on the left-hand side of Eq. (2.10), $O\left(\epsilon^{1 / 2}\right)$ term in the sum of $p_{3}^{r}$ and $p_{4}^{r}$ has to be zero. In fact, if one assumes particle 3 to be very energetic so that $E_{3}^{2}=\rho_{3} / \epsilon+\phi_{3}$, where $\rho_{3}(>0)$ and $\phi_{3}$ are constants, the $O\left(\epsilon^{1 / 2}\right)$ terms in the radial momentum conservation yield

$$
\sigma_{3} \sqrt{\rho_{3}\left[4\left(1-\delta_{(1)}\right)^{2}-1\right]}-2\left(1-\delta_{(1)}\right) \sqrt{\rho_{3}}=0 .
$$

There is no solution for $\rho_{3}$ under the assumption of $\rho_{3}>0$. By similar arguments, one can conclude that there is no energy extraction, expect for the case where particle 4 is very massive.

\section{CONCLUSION}

We have studied particle collision and energy-extraction efficiency, where a critical particle (particle 1) and subcritical particle (particle 2) collide near the event horizon of an extremal 
Kerr black hole and then two particles are produced, one of which escapes to infinity (particle 3) and another falls into the black hole (particle 4).

There is an upper limit of the energy of particle 3 , which is given by $E_{3, \max }=(2+\sqrt{3})^{2} E_{1}$. This is realized in the situation where particles 1 and 3 are massless, particle 3 is near critical, particle 1 is temporary outgoing and particles 2,3 and 4 are temporarily ingoing. The energyextraction efficiency, however, is bounded by 2.19 approximately, under the assumption that particle 4 has a mass of $O\left(\epsilon^{0}\right)$, where $\epsilon$ parametrizes the distance from the collision point to the horizon.

Since the CM energy of the near-horizon collision can be arbitrarily large, the collision can produce a heavy particle. From this viewpoint, we have considered the case in which the mass of particle 4 is of $O\left(\epsilon^{-1 / 2}\right)$. In this case, we found that the upper limit of the efficiency can indeed reach $(2+\sqrt{3})^{2} \simeq 13.9$. Thus, if the efficiency as large as 10 is observed for a collisional Penrose process, this strongly suggests the production of a heavy particle as a result of the collision of high CM energy.

Finally, let us give an example of collision with a high energy-extraction efficiency. We assume that particles 1 and 2 are protons, 3 is a photon, and 4 is some heavy particle and that the collision happens in the vicinity of the horizon with $\epsilon=10^{-8}$. Using $m_{1}=m_{2} \sim 1$

$\mathrm{GeV}$, we have $E_{\mathrm{cm}} \sim \sqrt{m_{1} m_{2} / \epsilon} \sim 10^{4} \mathrm{GeV}$. If $m_{4} \sim 10^{3} \mathrm{GeV}$, we have $\mu_{4} \sim m_{4}^{2} \epsilon \sim 10^{-2}$ $\mathrm{GeV}^{2}$ which is much smaller than $E_{1} E_{2}$. In this case, the efficiency is typically as large as 10.

Note added: While completing the current paper, the authors found that a paper [11] studying a similar problem appeared in arXiv. It will be interesting to compare the result in Ref. [11] with that in the current paper.

\section{ACKNOWLEDGMENTS}

The authors would like to thank T. Igata, M. Kimura, T. Kobayashi, K. Nakao, M. Patil and S. Yokoyama. This work was supported by JSPS KAKENHI Grant Numbers 26400282 (TH) and 15K05086 (UM). 


\section{Appendix A: Collision between a critical particle and an outgoing subcritical par-} ticle

Here we consider case $\mathrm{B}$, where $\sigma_{2}=1$ and particle 2 is subcritical. We can divide this case into the following three subcases: B1: $\sigma_{2}=\sigma_{4}=1\left(\sigma_{1}\right.$ and $\sigma_{3}$ can be either 1 or -1$)$. Particle 3 is critical. B2: $\sigma_{2}=\sigma_{3}=\sigma_{4}=1$. Both particles 3 and 4 are subcritical, and $2 E_{2}-\tilde{L}_{2}=\left(2 E_{3}-\tilde{L}_{3}\right)+\left(2 E_{4}-\tilde{L}_{4}\right)$. B3: $\sigma_{2}=\sigma_{3}=1, \sigma_{4}=-1$. Particle 3 is subcritical, and $2 E_{2}-\tilde{L}_{2}=2 E_{3}-\tilde{L}_{3}$, which implies particle 4 to be critical.

For case B1, the $O(\epsilon)$ terms in the radial momentum conservation yield

$$
\sigma_{1} \sqrt{3 E_{1}^{2}-m_{1}^{2}}-2 E_{1}+2 E_{3}\left(1-\delta_{(1)}\right)=\sigma_{3} \sqrt{E_{3}^{2}\left[4\left(\delta_{(1)}-1\right)^{2}-1\right]-m_{3}^{2}} .
$$

This is obtained from Eq. (3.1) after replacing $\sigma_{1}$ and $\sigma_{3}$ with $-\sigma_{1}$ and $-\sigma_{3}$, respectively. The equation obtained from the $O\left(\epsilon^{2}\right)$ terms of the radial momentum conservation is also the same as Eq. (3.2) after the above replacement. Therefore, $\eta_{\max }$ in this case is given by that in Sec. III A after changing the signs $\sigma_{i}(i=1,2,3,4)$. Note that the change of $\sigma_{3}$ requires the argument on the turning point, but one can finally see that this prescription is valid.

For case B2, the $O(\epsilon)$ terms in the radial momentum conservation equations yield

$$
\sigma_{1} \sqrt{3 E_{1}^{2}-m_{1}^{2}}-2 E_{1}=0
$$

This equation has no solution for $E_{1}$ under the assumption $E_{1}>0$ and $m_{1}^{2} \geq 0$. Thus, there is no energy extraction in this case.

For case B3, the $O(\epsilon)$ terms of the radial momentum conservation equations yield

$$
2 E_{1}-\sigma_{1} \sqrt{3 E_{1}^{2}-m_{1}^{2}}-2 E_{4}\left(1-\delta_{(1)}\right)=\sqrt{E_{4}^{2}\left[4\left(\delta_{(1)}-1\right)^{2}-1\right]-m_{4}^{2}} .
$$

Equation (A3) implies

$$
B_{1}-2 E_{4}\left(1-\delta_{(1)}\right)=\sqrt{E_{4}^{2}\left[4\left(\delta_{(1)}-1\right)^{2}-1\right]-m_{4}^{2}}
$$

for $\sigma_{1}=-1$ and $-\sqrt{B_{1}^{2}-m_{4}^{2}} \leq E_{4}<0$. From the forward-in-time condition and the argument on the turning point for a near-critical particle with negative energy, the restriction on $\delta_{(1)}$ is obtained as

$$
\delta_{(1)} \geq 1-\frac{\sqrt{E_{4}^{2}+m_{4}^{2}}}{2 E_{4}}
$$


Squaring the both sides of Eq. (A4) and then solving it for $E_{4}$, we obtain

$$
E_{4}=2\left(1-\delta_{(1)}\right) B_{1}+\sqrt{B_{1}^{2}\left[4\left(\delta_{(1)}-1\right)^{2}-1\right]-m_{4}^{2}} .
$$

This implies the lower limit of $E_{4}$ is given by

$$
E_{4, \min }=-(2+\sqrt{3}) E_{1},
$$

where $m_{1}=m_{4}=0$ and $\delta_{(1)}=3 / 2$ hold. The $O\left(\epsilon^{2}\right)$ terms of the radial momentum conservation equation yield

$$
\begin{array}{r}
-\sigma_{1} \frac{E_{1}^{2}}{\sqrt{3 E_{1}^{2}-m_{1}^{2}}}+\frac{\left(3 E_{2}-\tilde{L}_{2}\right)\left(E_{2}-\tilde{L}_{2}\right)-m_{2}^{2}}{2\left(2 E_{2}-\tilde{L}_{2}\right)}=\frac{E_{4}^{2}\left[1-4\left(2 \delta_{(1)}-\delta_{(2)}\right)\left(1-\delta_{(1)}\right)\right]}{\sqrt{E_{4}^{2}\left[4\left(\delta_{(1)}-1\right)^{2}-1\right]-m_{4}^{2}}} \\
+\frac{2 E_{2}-\tilde{L}_{2}}{2}-2 E_{4}\left(2 \delta_{(1)}-\delta_{(2)}\right)-\frac{\left(E_{1}+E_{2}-E_{4}\right)^{2}+m_{3}^{2}}{2\left(2 E_{2}-\tilde{L}_{2}\right)} .
\end{array}
$$

The lower limit of $E_{4}$ can be realized only for $m_{1}=m_{4}=0$ and $\delta_{(1)}=3 / 2$. Because the denominator of the first term on the right-hand side of Eq. (A8) becomes zero when $m_{4}=0$ and $\delta_{(1)}=3 / 2$, we need $1-4\left(2 \delta_{(1)}-\delta_{(2)}\right)\left(1-\delta_{(1)}\right)=0$, which is possible when $\delta_{(2)}=7 / 2$. Therefore, the upper limit of the energy-extraction efficiency in this case is given by

$$
\eta_{\max }=1-\frac{E_{4, \min }}{E_{1}+E_{2}}=\frac{(3+\sqrt{3}) E_{1}+E_{2}}{E_{1}+E_{2}}<3+\sqrt{3},
$$

where we have assumed $m_{1}=m_{4}=0, \delta_{(1)}=3 / 2$, and $\delta_{(2)}=7 / 2$.

If we assume $m_{4}=O\left(\epsilon^{-1 / 2}\right)$, the energy-extraction efficiency can be larger as in case A. For case $\mathrm{B} 1$, the result is the same as for case $\mathrm{A}$ after replacing $\sigma_{i}$ with $-\sigma_{i}(i=1,2,3,4)$. Therefore, we can obtain the upper limit $\simeq 13.9$, only if a very massive particle is produced to fall into the black hole. For cases B2 and B3, the energy extraction is quite modest. Namely, the efficient energy extraction is not realized even when an subcritical outgoing particles is considered, as long as its counterpart is critical.

[1] M. Bañados, J. Silk, and S. M. West, "Kerr Black Holes as Particle Accelerators to Arbitrarily High Energy", Phys. Rev. Lett. 103 (2009) 111102 [arXiv:0909.0169 [hep-ph]].

[2] T. Harada and M. Kimura, "Black holes as particle accelerators: a brief review," Class. Quant. Grav. 31 (2014) 243001 [arXiv:1409.7502 [gr-qc]].

[3] T. Piran, J. Shaham, and J. Katz, "High efficiency of the Penrose mechanism for particle collisions", Astrophys. J. 196 (1975) L107. 
[4] T. Piran and J. Shaham, "Upper bounds on collisional Penrose processes near rotating blackhole horizons", Phys. Rev. D 16 (1977) 1615.

[5] T. Harada, H. Nemoto, and U. Miyamoto, "Upper limits of particle emission from high-energy collision and reaction near a maximally rotating Kerr black hole", Phys. Rev. D 86 (2012) 024027 [arXiv:1205.7088 [gr-qc]].

[6] J. D. Schnittman, "A revised upper limit to energy extraction from a Kerr black hole", Phys. Rev. Lett. 113 (2014) 261102. [arXiv:1410.6446 [gr-qc]].

[7] E. Berti, R. Brito and V. Cardoso, "Ultrahigh-energy debris from the collisional Penrose process," Phys. Rev. Lett. 114 (2015) 25, 251103 [arXiv:1410.8534 [gr-qc]].

[8] E. Leiderschneider and T. Piran, "Super-Penrose collisions are inefficient - a Comment on: Black hole fireworks: ultra-high-energy debris from super-Penrose collisions", arXiv:1501.01984 [gr-qc].

[9] T. Harada and M. Kimura, "Collision of two general geodesic particles around a Kerr black hole," Phys. Rev. D 83 (2011) 084041 [arXiv:1102.3316 [gr-qc]].

[10] T. Harada and M. Kimura, "Collision of an innermost stable circular orbit particle around a Kerr black hole," Phys. Rev. D 83 (2011) 024002 [arXiv:1010.0962 [gr-qc]].

[11] E. Leiderschneider and T. Piran, "On the maximal efficiency of the collisional Penrose process," arXiv:1510.06764 [gr-qc]. 